\title{
ATLAS diboson excess from low scale supersymmetry breaking
}

\author{
Christoffer Petersson ${ }^{a, b, c}$ and Riccardo Torre $^{d}$ \\ ${ }^{a}$ Department of Fundamental Physics, Chalmers University of Technology, \\ 412 96 Göteborg, Sweden \\ ${ }^{b}$ Physique Théorique et Mathématique, Université Libre de Bruxelles, \\ C.P. 231, 1050 Brussels, Belgium \\ ${ }^{c}$ International Solvay Institutes, \\ 1050 Brussels, Belgium \\ ${ }^{d}$ Dipartimento di Fisica e Astronomia, Università di Padova and INFN Sezione di Padova, \\ Via Marzolo 8, I-35131 Padova, Italy \\ E-mail: christoffer.petersson@chalmers.se, riccardo.torre@cern.ch
}

ABSTRACT: We provide an interpretation of the recent ATLAS diboson excess in terms of a class of supersymmetric models in which the scale of supersymmetry (SUSY) breaking is in the few $\mathrm{TeV}$ range. The particle responsible for the excess is the scalar superpartner of the Goldstone fermion associated with SUSY breaking, the sgoldstino. This scalar couples strongly to the Standard Model vector bosons and weakly to the fermions, with all coupling strengths determined by ratios of soft SUSY breaking parameters over the SUSY breaking scale. Explaining the ATLAS excess selects particular relations and ranges for the gaugino masses, while imposing no constraints on the other superpartner masses. Moreover, this signal hypothesis predicts a rate in the $Z \gamma$ final state that is expected to be observable at the LHC Run II already with a few $\mathrm{fb}^{-1}$ of integrated luminosity.

KeYwords: Supersymmetry Phenomenology

ARXIV EPRINT: 1508.05632 


\section{Contents}

1 Introduction 1

2 The sgoldstino model $\quad 2$

3 Explaining the diboson excess 4

4 Other analyses $\quad 8$

$\begin{array}{llr}5 & \text { Conclusions } & 9\end{array}$

\section{Introduction}

The ATLAS Collaboration recently published a search for resonances in the boson tagged di-jet mass distribution, featuring an excess of events around $2 \mathrm{TeV}$ [1]. Despite the fact that the statistical significance of the excess (up to $3.4 \sigma$ locally and $2.5 \sigma$ globally) is limited, the appearance of other excesses, though less significant, in similar final states and in the same mass region, such as in the CMS search in ref. [2], motivates some theoretical effort to understand the possible origin of these fluctuations. Several papers have already appeared, aimed at explaining the excess in terms of different new physics models [3-37] and scrutinizing the ATLAS analysis [38].

In the context of supersymmetry (SUSY), it is not straightforward to find an explanation of this excess. For instance, with the usual particle content of the minimal SUSY extension of the Standard Model (MSSM), there is no candidate particle that could give rise to such a signal. However, in the case where SUSY is broken at a low scale, ${ }^{1}$ additional degrees of freedom, related to the spontaneous breaking of SUSY, are present and can become phenomenologically relevant. In particular, the Goldstone fermion of SUSY breaking, the goldstino, and, when SUSY is linearly realized, its scalar superpartner, the sgoldstino, can couple strongly to some of the SM particles. The interaction strengths of the goldstino and sgoldstino are determined by ratios of the usual soft SUSY breaking parameters of the MSSM over the supersymmetry breaking scale.

In this paper we provide an interpretation of the ATLAS diboson excess in terms of a class of SUSY models where the SUSY breaking scale is in the few $\mathrm{TeV}$ range, with a $2 \mathrm{TeV}$ sgoldstino scalar being responsible for the excess. For different discussions concerning sgoldstino physics, see, for example, refs. [40-51]. The sgoldstino couples mostly to the SM vector bosons, with interaction strengths determined by ratios of the gaugino masses over the SUSY breaking scale, whereas its couplings to the SM fermions are generically

\footnotetext{
${ }^{1}$ The current experimental lower bound on the SUSY breaking scale is at or below $1 \mathrm{TeV}$ [39] (the exact value depends on the superpartner spectrum).
} 
suppressed. In what follows, we study the compatibility of this signal hypothesis with the excess, identify the relevant region of the parameter space (in terms of the gaugino masses and the SUSY breaking scale) and discuss the relations to other searches in correlated channels, such as $\gamma \gamma$ and $Z \gamma$.

The paper is organized as follows. In section 2 we provide the sgoldstino couplings to the SM and in section 3 we extract the relevant values for the gaugino masses and SUSY breaking scale that allow us to explain the ATLAS excess. We describe the constraints from, and implications for, other searches in section 4 and conclude in section 5 .

\section{The sgoldstino model}

If SUSY is realized in Nature, since the SM particles are not mass-degenerate with their superpartners, it must be in a broken phase at low energies. A general consequence of the spontaneous breaking of (global) SUSY is the existence of a Goldstone fermion, the goldstino. We will assume that the goldstino resides in a gauge singlet chiral superfield, with SUSY linearly realized,

$$
X=x+\sqrt{2} \theta \widetilde{G}+\theta^{2} F_{X}
$$

where the auxiliary field acquires a vacuum expectation value (VEV), $\left\langle F_{X}\right\rangle=f$, that gives the dominant contribution to SUSY breaking. The focus of this paper will be on the complex scalar superpartner of the goldstino, the sgoldstino $x$ in eq. (2.1). In contrast to the goldstino, the sgoldstino is not protected by the Goldstone theorem and therefore it will generically acquire a mass, with a value that is model-dependent. Also, in general, the masses of the CP-even and CP-odd scalars do not need to be equal [52]. Here we assume them to be equal and fix them to be $2 \mathrm{TeV}$.

One way to take into account the interactions of the goldstino and sgoldstino is to simply promote all the usual MSSM soft terms to SUSY operators involving the goldstino superfield in eq. (2.1). For instance, the gaugino masses $m_{i}$, where $i=1,2$ and 3 corresponds to the bino, wino and gluino masses, respectively, are promoted to the following SUSY operators,

$$
\frac{m_{i}}{2} \lambda_{(i)}^{\alpha} \lambda_{(i) \alpha} \rightarrow \frac{m_{i}}{2 f} \int d^{2} \theta X W_{(i)}^{\alpha} W_{(i) \alpha},
$$

where $W_{(i)}^{\alpha}$, for $i=1,2$ and 3 , corresponds to the gauge field-strength superfield for $\mathrm{U}(1)_{Y}$, $\mathrm{SU}(2)_{L}$ and $\mathrm{SU}(3)_{c}$. Note that by taking the auxiliary component of $X$ and inserting its VEv, $\left\langle F_{X}\right\rangle=f$, one recovers the usual gaugino mass terms. The goldstino or sgoldstino interactions are obtained by taking the fermion or scalar component of $X$.

We will from hereon focus on the interactions of the sgoldstino $x=(\phi+i a) / \sqrt{2}$, where $\phi$ and $a$ are the $\mathrm{CP}$-even and $\mathrm{CP}$-odd real scalar components. All the relevant vertices arising from eq. (2.2) can now be collected and included in the following sgoldstino Lagrangian [40]

$$
\mathcal{L}=\mathcal{L}_{g g}+\mathcal{L}_{\gamma \gamma}+\mathcal{L}_{Z \gamma}+\mathcal{L}_{Z Z}+\mathcal{L}_{W W}+\mathcal{L}_{G G}
$$

where

$$
\mathcal{L}_{g g}=\frac{m_{3}}{2 \sqrt{2} f}\left(-\phi G^{a \mu \nu} G_{\mu \nu}^{a}+a G^{a \mu \nu} \widetilde{G}_{\mu \nu}^{a}\right)
$$




$$
\begin{aligned}
\mathcal{L}_{W W} & =\frac{m_{2}}{\sqrt{2} f}\left(-\phi W^{+\mu \nu} W_{\mu \nu}^{-}+a W^{+\mu \nu} \widetilde{W}_{\mu \nu}^{-}\right), \\
\mathcal{L}_{Z Z} & =\frac{m_{1} s_{\theta_{W}}^{2}+m_{2} c_{\theta_{W}}^{2}}{2 \sqrt{2} f}\left(-\phi Z^{\mu \nu} Z_{\mu \nu}+a Z^{\mu \nu} \widetilde{Z}_{\mu \nu}\right), \\
\mathcal{L}_{\gamma \gamma} & =\frac{m_{1} c_{\theta_{W}}^{2}+m_{2} s_{\theta_{W}}^{2}}{2 \sqrt{2} f}\left(-\phi F^{\mu \nu} F_{\mu \nu}+a F^{\mu \nu} \widetilde{F}_{\mu \nu}\right), \\
\mathcal{L}_{Z \gamma} & =\frac{\left(m_{2}-m_{1}\right) s_{\theta_{W}} c_{\theta_{W}}}{\sqrt{2} f}\left(-\phi F^{\mu \nu} Z_{\mu \nu}+a F^{\mu \nu} \widetilde{Z}_{\mu \nu}\right), \\
\mathcal{L}_{G G} & =\frac{m_{\phi}^{2}}{2 \sqrt{2} f}(-\phi \widetilde{G} \widetilde{G}+i a \widetilde{G} \widetilde{G})+\text { h.c. },
\end{aligned}
$$

where $s_{\theta_{W}}=\sin \theta_{W}$ and $c_{\theta_{W}}=\cos \theta_{W}$, with $\theta_{W}$ being the weak mixing angle, and the tilde denotes e.g. $\widetilde{G}_{\mu \nu}^{a}=(1 / 2) \epsilon_{\mu \nu \rho \sigma} G^{a \rho \sigma}$. The interactions in eq. (2.4f) arise from the operator $m_{\phi}^{2} /\left(4 f^{2}\right)\left(X^{\dagger} X\right)^{2}$ in the Kahler potential, from which also the soft mass $m_{\phi}=m_{a}$ for the $\mathrm{CP}$-even and CP-odd sgoldstino scalars $\phi$ and $a$ arises. Notice that the sgoldstino couples purely to the transverse components of the $W$ and $Z$ bosons. A small coupling to the longitudinal components can arise through mixing with the Higgs, but for the region of parameter space that we consider such a mixing is negligible.

From the sgoldstino Lagrangian (2.3) we can compute the partial decay widths for the sgoldstino scalar $\phi$ (the corresponding widths for $a$ are obtained by simply replacing $\phi \rightarrow a$ since they are identical to those of $\phi$ ),

$$
\begin{aligned}
\Gamma(\phi \rightarrow g g) & =\frac{m_{3}^{2} m_{\phi}^{3}}{4 \pi f^{2}}, \\
\Gamma(\phi \rightarrow W W) & =\frac{m_{2}^{2} m_{\phi}^{3}}{16 \pi f^{2}} k\left(\frac{m_{W}}{m_{\phi}}\right), \\
\Gamma(\phi \rightarrow Z Z) & =\frac{\left(m_{1} s_{\theta_{W}}^{2}+m_{2} c_{\theta_{W}}^{2}\right)^{2} m_{\phi}^{3}}{32 \pi f^{2}} k\left(\frac{m_{Z}}{m_{\phi}}\right), \\
\Gamma(\phi \rightarrow \gamma \gamma) & =\frac{\left(m_{1} c_{\theta_{W}}^{2}+m_{2} s_{\theta_{W}}^{2}\right)^{2} m_{\phi}^{3}}{32 \pi f^{2}}, \\
\Gamma(\phi \rightarrow Z \gamma) & =\frac{\left(m_{2}-m_{1}\right)^{2} s_{\theta_{W}}^{2} c_{\theta_{W}}^{2} m_{\phi}^{3}}{16 \pi f^{2}}\left(1-\frac{m_{Z}^{2}}{m_{\phi}^{2}}\right)^{3}, \\
\Gamma(\phi \rightarrow G G) & =\frac{m_{\phi}^{5}}{32 \pi f^{2}},
\end{aligned}
$$

where the function $k(x)=\left(1-4 x^{2}+6 x^{4}\right)\left(1-4 x^{2}\right)^{1 / 2}$ is close to unity in the case where $m_{W}, m_{Z} \ll m_{\phi}=2 \mathrm{TeV}$.

The interactions between the sgoldstino and the SM fermions arise from superpotential operators such as $\left(A_{u} / f\right) X Q H_{u} U^{c}$, which, upon taking the auxiliary component of $X$ and inserting its VEv, also give rise to the usual $A$-terms. Since we are requiring all soft parameters to be smaller than $\sqrt{f}$, the sgoldstino couplings will be suppressed at least by the ratio of the Higgs VEV over $\sqrt{f}$, which makes the sgoldstino decays to SM fermions negligible with respect to the sgoldstino decays to vector bosons. 


\begin{tabular}{|c|c|c|c|}
\hline $\begin{array}{l}\text { selection region } \\
\text { final state }\end{array}$ & $W W$ & $W Z$ & $Z Z$ \\
\hline$W W$ & 0.39 & 0.37 & 0.16 \\
\hline$W Z$ & 0.33 & 0.44 & 0.25 \\
\hline$Z Z$ & 0.27 & 0.47 & 0.37 \\
\hline
\end{tabular}

Table 1. Efficiencies $\epsilon_{V_{f} V_{f}^{\prime} \rightarrow V_{s} V_{s}^{\prime}}$ for a final state $V_{f} V_{f}^{\prime}$ to end up in the $V_{s} V_{s}^{\prime}$ selection region.

The sgoldstino is produced at the LHC by gluon-gluon fusion with the leading order production cross section [41] (summing the two equal contributions from the CP-even scalar $\phi$ and the CP-odd scalar $a$, with $m_{\phi}=m_{a}$ )

$$
\sigma=\frac{\pi^{2} \Gamma(\phi \rightarrow g g)}{4 s m_{\phi}} \times \int_{\frac{m_{\phi}^{2}}{s}}^{1} \frac{d x}{x} f_{p / g}\left(x, m_{\phi}^{2}\right) f_{p / g}\left(\frac{m_{\phi}^{2}}{x s}, m_{\phi}^{2}\right),
$$

where the partial width $\Gamma(\phi \rightarrow g g)$ is given by eq. $(2.5 \mathrm{a}), s$ is the center of mass energy squared and $f_{p / g}\left(x, Q^{2}\right)$ are the parton distribution functions defined at the scale $Q^{2}$. Since $\Gamma_{\phi} / m_{\phi}$ is below $10 \%$ for the sgoldstino in the relevant region of the parameter space, eq. (2.6), which assumes the narrow width approximation, is always reliable.

\section{Explaining the diboson excess}

In this section, to assess the compatibility of a sgoldstino signal with the ATLAS diboson excess, we compare the number of signal events the sgoldstino gives rise to with the number of excess events reported by ATLAS. Figure 5 of ref. [1] shows the invariant mass distribution of the boson tagged jets for the $W Z, W W$ and $Z Z$ selection regions (SRs). These regions have large overlaps due to the overlap of the shapes of the single $W$ and $Z$ tagged jet mass distributions. We take into account this overlap by computing the different efficiencies $\epsilon_{V_{f} V_{f}^{\prime} \rightarrow V_{s} V_{s}^{\prime}}$ for a final state $V_{f} V_{f}^{\prime}$ to end up in the $V_{s} V_{s}^{\prime} \mathrm{SR}$. The values of these efficiencies are given in table 1.

We consider the window $1.75-2.25 \mathrm{TeV}$ in the boson tagged dijet mass distribution, around the mass hypothesis, and compare the number of observed events with the number of events predicted by the SM. This approach is quite conservative, since it combines the higher significance of the central bins with the lower significance of the side bins. We follow this approach because it better accounts both for a finite width of the resonance and for shower and reconstruction effects that smear the resonance peak (see the simulated signal shapes in figure 5 of ref. [1] for a quantitative assessment of these effects). To estimate the error on the number of excess events that we count in the considered window we proceed as follows: the Poissonian central $68 \%$ CL interval corresponding to the total number of observed events in the window would give an over-estimate of the error, giving rise to a local statistical significance that is below $2 \sigma$, i.e. much less than the one reported by ATLAS. To give justice to the higher statistical significance observed by ATLAS we estimate the error on the excess events as the Poissonian central 68\% CL interval on the number of excess 
events. Though statistically incorrect, this procedure gives a statistical significance that better matches the one reported by ATLAS.

A reliable combination of the three SRs $W Z, W W$ and $Z Z$ would require detailed knowledge of the degree of correlation between these three channels that go beyond the effect of the efficiencies $\epsilon$ that we take into account, such as the correlation of all the systematic uncertainties. Since we do not have this information at our disposal, we instead extract the signal from a single channel and then confront it with the number of events observed in the other two channels, as well as with the other relevant analyses.

Our model predicts the largest production rate in the $W W$ channel and therefore, to also minimize the uncertainties coming from our extraction of the tagging efficiencies, we extract the sgoldstino signal yield from the $W W$ SR. For an invariant mass of $2 \mathrm{TeV}$, we obtain from the ATLAS analysis $S_{W W}=4.2_{-2.0}^{+3.2}$ excess events in the considered window, where the error band represents Poissonian central 68\% CL interval, which is what we refer to as $1 \sigma$ interval throughout the paper. The number of signal events produced by the sgoldstino in the various $V_{s} V_{s}^{\prime}$ SRs is given by

$$
\begin{aligned}
S_{V_{s} V_{s}^{\prime}}=\left[\sigma \times \mathrm{BR}_{W W} \times \mathcal{A}_{W W} \times \mathrm{BR}_{W W \rightarrow \text { had }} \times \epsilon_{W W \rightarrow V_{s} V_{s}^{\prime}}\right. & \\
& \left.+\sigma \times \mathrm{BR}_{Z Z} \times \mathcal{A}_{Z Z} \times \mathrm{BR}_{Z Z \rightarrow \text { had }} \times \epsilon_{Z Z \rightarrow V_{s} V_{s}^{\prime}}\right] \mathcal{L},
\end{aligned}
$$

where $\mathrm{BR}_{i j}$ corresponds to the sgoldstino decay branching ratio into the $i j$ final state, the factors $\mathcal{A}_{W W}$ and $\mathcal{A}_{Z Z}$ are the acceptances for the kinematic and topology selections and include the signal acceptance to the invariant mass cut in the window we consider, $\mathrm{BR}_{W W \rightarrow \text { had }}$ and $\mathrm{BR}_{Z Z \rightarrow \text { had }}$ are the hadronic branching ratios of the $W W$ and $Z Z$ channels, respectively, and $\mathcal{L}=20.3 \mathrm{fb}^{-1}$ is the integrated luminosity.

The knowledge of the acceptance factors is a key ingredient to estimate the number of events starting from a certain theoretical value of $\sigma \times \mathrm{BR}$. Unfortunately, the ATLAS analysis only reports the value of these acceptances for a vector, and a spin-two signal hypothesis, the bulk graviton, that decays into longitudinally polarized vector bosons. This information does not allow us to extract the acceptance for a scalar particle decaying to transverse vector bosons, as is the case of the sgoldstino. Comparing the ATLAS acceptances with the ones that CMS reports in ref. [2], which is the counterpart of ref. [1], where also a spin-two particle decaying to transverse vector bosons is considered, the RS graviton, we expect the acceptances for a resonance decaying into transverse gauge bosons to be about $50 \%$ smaller than the acceptances for a resonance decaying into longitudinal vectors, which is the case reported by ATLAS. Such a reduction in the acceptances would require a larger $\sigma \times$ BR to explain the ATLAS excess, thereby selecting a region of the parameter space with lower values of the relevant parameters, namely $m_{2}, m_{3}$ and $\sqrt{f}$. However, since we cannot reliably estimate this number we will instead use the acceptances that ATLAS reports for the bulk graviton also for the sgoldstino. This also allows us, in a more consistent way, to compare with other analyses where the same spin-two signal hypothesis is considered. In fact, under the assumption that the ratio of the acceptances of our resonance over the bulk graviton is approximately constant for the different analyses, the error that we make when comparing the extracted cross section for our resonance with the 
other relevant constraints should approximately cancel out. Nevertheless, this represents the main source of uncertainty in our estimation of the cross section. For this reason we stress that, in the case in which a higher significance of this excess is observed in Run II, it is of primary importance that the experimental collaborations provide the acceptances for all the relevant spin hypotheses and polarizations of the final state vector bosons.

The acceptance factors in eq. (3.1) are estimated starting from the total selection efficiencies reported in figure 2 (b) of ref. [1], divided by the aforementioned boson tagging efficiencies in the respective SRs. This number is then multiplied by the efficiency corresponding to the invariant mass cut in the window we consider, estimated from the signal shape reported by ATLAS in the $W W$ SR for the spin-two resonance in figure 5 (b), which is the one with more available statistics and which is about 0.87 . The resulting two acceptances in eq. (3.1) are, as expected, almost identical, $\mathcal{A}_{W W}=0.22$ and $\mathcal{A}_{Z Z}=0.21$.

The parameters that most strongly affect the diboson channels relevant for the excess are $m_{2}, m_{3}$ and $\sqrt{f}$, while in the case of the $\gamma \gamma$ and $Z \gamma$ channels, there is also some dependence on $m_{1}$. The ATLAS search in the $\gamma \gamma$ channel of ref. [53] place a 95\% CL upper limit on $\sigma \times \mathrm{BR}_{\gamma \gamma}$ at around $0.3 \mathrm{fb}$ for a mass of $2 \mathrm{TeV}$. We stress that also this result is obtained assuming a spin-two resonance and can not be straightforwardly used to constrain a scalar. However, since we do not expect huge changes in the efficiencies, this gives us an estimate of the current bound on a heavy scalar decaying to $\gamma \gamma$. As can be seen from eq. (2.5d), this bound can be completely evaded by choosing $m_{1} \approx-m_{2} \tan ^{2} \theta_{W}$ since, in this case, $\mathrm{BR}_{\gamma \gamma} \approx 0$. However, since we find that there is a wide range of $m_{1}$ that satisfies the $\gamma \gamma$ constraint, without affecting the diboson channels, we choose not to fix any particular relation to $m_{2}$, but instead we set it to a reference value, $m_{1}=100 \mathrm{GeV},{ }^{2}$ for which the constraint from the $\gamma \gamma$ search is satisfied in the entire range or $m_{2}, m_{3}$ and $\sqrt{f}$ that we consider.

The regions enclosed by the solid grey curves in figure 1 represent the regions in the $\left(m_{3}, \sqrt{f}\right)$ plane where the $2 \mathrm{TeV}$ sgoldstino signal reproduces the number of excess events observed by ATLAS in the $W W$ SR in the invariant mass window we consider, within the $1 \sigma$ band, namely $S_{W W}=4.2_{-2.0}^{+3.2}$. The four different regions correspond to four representative values of $m_{2}$, namely $1,2.5,4$ and $5 \mathrm{TeV}$. Of course, different values of $m_{2}$ interpolate between these regions covering a large part of the $m_{3}<\sqrt{f}$ plane. In figure 1 we have also imposed the constraint $m_{2}, m_{3}<\sqrt{f}$ to ensure a valid expansion in eq. (2.3) in terms of effective operators. The current experimental limits on $m_{3}$ and $\sqrt{f}$ depend on the masses of the other superpartners on which the fit to the ATLAS excess imposes no constraints. In figure 1 , we take a conservative approach and require both $m_{3}$ and $\sqrt{f}$ to be above 1.5 TeV, corresponding to the most stringent current bound on both the gluino mass [55] and the SUSY breaking scale [39].

In the four regions shown in figure 1 , the predicted values for the cross sections in the other two diboson channels span the ranges $\sigma \times \mathrm{BR}_{W Z} \in[2.3,7.6] \mathrm{fb}$ and the $\sigma \times$ $\mathrm{BR}_{Z Z} \in[0.7,2.2] \mathrm{fb}$. For these cross section intervals, by using eq. (3.1), we compute the $1 \sigma$

\footnotetext{
${ }^{2}$ Notice that when $m_{1}<m_{\phi} / 2$ the sgoldstino can decay into two neutralinos with a coupling that is generally model dependent. While this decay is not expected to significantly affect the region of the parameter space where the model reproduces the ATLAS excess, the final states it can give rise to, e.g. two photons and missing energy, are potentially interesting at the LHC.
} 


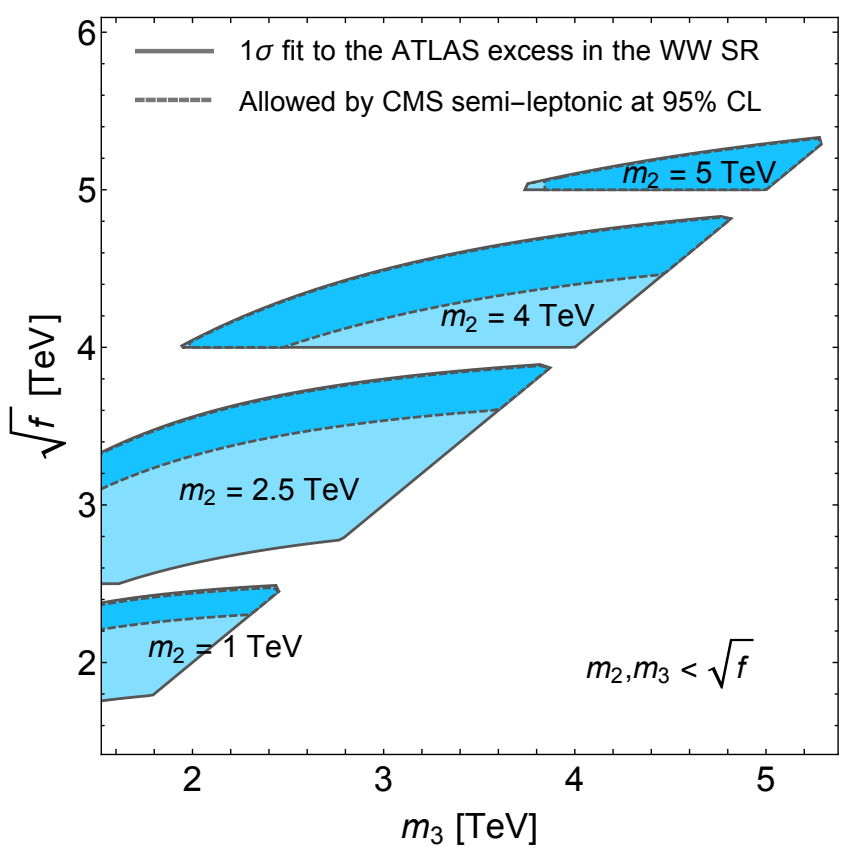

Figure 1. The four regions enclosed by the solid lines, corresponding to four different values of $m_{2}$, show the points in the plane $\left(m_{3}, \sqrt{f}\right)$ for which a $2 \mathrm{TeV}$ sgoldstino can explain the ATLAS diboson excess [1] within $1 \sigma$. The (dark blue) regions enclosed by the dashed lines correspond to the subset of these point that satisfies the 95\% CL limit placed by the semi-leptonic diboson search by CMS [54].

intervals for the number of excess events predicted in the $W Z$ and $Z Z$ SRs, and we obtain $S_{W Z} \in[2.4,8.0]$ and $S_{Z Z} \in[1.3,4.2]$. Comparing these numbers with the corresponding values extracted from the ATLAS analysis, namely $7.0_{-2.6}^{+3.8}$ and $6.4_{-2.4}^{+3.6}$, respectively, we see that the $W Z \mathrm{SR}$ is well within the statistical $1 \sigma$ band, while $Z Z$ shows a slight tension. However, this tension is removed once one includes systematic uncertainties, which are at the level of $50 \%$ for the signal [1]. Nevertheless, it is worth asking if we can directly understand this tension from the ATLAS analysis.

One would expect that, at such high invariant masses, the shapes of the invariant mass distributions in the three SRs $W W, W Z$ and $Z Z$ should be very similar. ${ }^{3}$ However, in the ATLAS analysis, while the shapes in the $W W$ and $W Z$ SRs are indeed very similar, the one in the $Z Z$ SR looks qualitatively different, as it falls off much faster. In order to assess the possible origin of the tension that we find between the signal in the $Z Z \mathrm{SR}$ and in the other two SRs, we compute the number of excess events in the $Z Z$ SR that would be obtained if we instead use the $W Z$ or the $W W$ shape, with the $Z Z$ normalization, as $Z Z$ background distribution. With the $W Z$ or the $W W$ background distribution shapes, we obtain only 3.0 and 1.7 excess events, respectively, in the window we consider, to be compared with the 6.4 obtained from the ATLAS fit to the background distribution in the

\footnotetext{
${ }^{3}$ It is well-known that QCD jets fake $W$ jets easier than $Z$ jets, which results in a larger background for the $W W$ and $W Z$ signal regions than for the $Z Z$ signal region. However, we do not expect this to significantly affect the shapes of the boson-tagged dijet mass distributions in the different signal regions.
} 
$Z Z \mathrm{SR}$. This shows that the tension in the $Z Z$ channel could be a consequence of the smaller statistics in this channel.

\section{Other analyses}

Now that we have extracted the interesting region of the parameter space of the sgoldstino that allows us to reproduce the excess of events observed by ATLAS, we confront our signal hypothesis with the other relevant searches. The first search to compare with is reported in ref. [2] and is the CMS analogous of the ATLAS fully hadronic search [1]. This sets the limits (for the same spin and polarization hypothesis that is considered by ATLAS and that we used to extract the signal) $\sigma \times \mathrm{BR}_{W W}<11 \mathrm{fb}$ and $\sigma \times \mathrm{BR}_{Z Z}<10 \mathrm{fb}$ for a $2 \mathrm{TeV}$ mass hypothesis, which lie above our $1 \sigma$ bands for the corresponding quantities and hence do not set any further constraint on the allowed parameter space.

The analysis that sets the strongest constraint on the $\sigma \times \mathrm{BR}$ for a $2 \mathrm{TeV}$ resonance decaying to gauge bosons is a CMS search in the semi-leptonic channel with either 1 lepton ( $W W$ channel) or 2 leptons ( $Z Z$ channel) [54]. There is no overlap in this case due to the selection with different numbers of leptons. We can therefore directly compare our predicted $\sigma \times \mathrm{BR}$ with the limits this search places in the respective channels, $\sigma \times \mathrm{BR}_{W W}<3 \mathrm{fb}$ and $\sigma \times \mathrm{BR}_{Z Z}<8 \mathrm{fb}$ at $2 \mathrm{TeV}$. While the $Z Z$ bound does not constrain our parameter space, we get a constraint from the $W W$ channel, which reduces the allowed parameter space in the $\left(m_{3}, \sqrt{f}\right)$ plane in figure 1 to the (dark blue) regions enclosed by the dashed lines. The (light blue) regions that remain outside the dashed contours are excluded at 95\% CL by the CMS semi-leptonic analysis [54] in the $W W$ channel.

Given the fact that the dominant decay mode of the sgoldstino is into two gluons, one expects a contribution in the dijet channel. We can do a simple estimate of dijet rate using eqs. (2.5a) and (2.5b), from which it follows that $\mathrm{BR}_{g g} / \mathrm{BR}_{W W}=4\left(m_{3} / m_{2}\right)^{2}$. In order to fit the diboson excess, without being excluded by the semileptonic searches, we got that $\sigma \times \mathrm{BR}_{W W} \sim 3 \mathrm{fb}$, implying that $\sigma \times \mathrm{BR}_{g g} \sim 12\left(m_{3} / m_{2}\right)^{2} \mathrm{fb}$. The largest dijet rate is achieved in the lower left parameter space region of figure 1 , where the ratio $m_{3} / m_{2}$ is maximimal, around 2.4. Thus, the maximum dijet rate is around $70 \mathrm{fb}$, which is below the current limit $[39,56]$, but could possibly account for the slight excess of events observed by CMS around $1.8 \mathrm{TeV}$ of invariant mass [56].

Let us finally comment on other possible interesting channels. If the ATLAS diboson excess is caused by the scalar sgoldstino, no signal is expected in the $Z H$ and $W H$ channels. Hence, if statistically significant excesses are found in Run II in these channels, it would point toward other new physics scenarios. Instead, the most relevant other channels for the sgoldstino signal hypothesis are the $\gamma \gamma$ and $Z \gamma$ channels. As was discussed in the previous section, the bound from existing $\gamma \gamma$ searches can always be satisfied by choosing $m_{1}$ to be within a rather wide range around the value $m_{1} \approx-m_{2} \tan ^{2} \theta_{W}$, for which $\mathrm{BR}_{\gamma \gamma}$ vanishes, as can be seen from eq. (2.5d). Clearly, the allowed range of $m_{1}$ is wider for larger values of $m_{2}$.

One way to place a constraint on $m_{1}$ would be to search for a resonance in the $Z \gamma$ channel at $2 \mathrm{TeV}$, and use the relation between $m_{1}$ and $m_{2}$ in eq. (2.5e). The only search in 
the $Z \gamma$ channel that we are aware of is the ATLAS analysis in ref. [57] which only extends to invariant masses up to $1.6 \mathrm{TeV}$. Therefore we do not get any constraint from this search. However, it is interesting to note that the exclusion at $1.6 \mathrm{TeV}$ is $\sigma \times \mathrm{BR}_{Z \gamma}<2.4 \mathrm{fb}$ for a scalar. We find that, in a large part of the parameter space that explains the ATLAS diboson excess, and for a wide range of values of $m_{1}$ within the region allowed by $\gamma \gamma$ searches, the $\sigma \times \mathrm{BR}_{Z \gamma}$ we get for the $2 \mathrm{TeV}$ sgoldstino is of the order of a few $\mathrm{fb}$. This suggests that once the $Z \gamma$ search is extended to include $2 \mathrm{TeV}$ invariant masses, which will be possible at the $13 \mathrm{TeV}$ LHC Run II with a few inverse fb of integrated luminosity, this channel will be sensitive to the sgoldstino signal and could quickly lead to a discovery or exclude most of the parameter space presently allowed.

\section{Conclusions}

In this paper we have provided an explanation of the recently reported ATLAS diboson excess in terms of a $2 \mathrm{TeV}$ sgoldstino scalar, which is present in a class of supersymmetric models in which the supersymmetry breaking scale is in the few $\mathrm{TeV}$ range. Fitting this excess selects particular ranges and relations among the gaugino masses, while imposing no constraints on the other superpartner masses. In terms of other resonance searches, while no signal is expected in the $Z H$ and $W H$ channels, we expect the most sensitive channel to be $Z \gamma$.

The sgoldstino production cross-section, which originates from gluon-gluon fusion, is expected to increase by a factor of about 19 when going from $\sqrt{s}=8 \mathrm{TeV}$ to $13 \mathrm{TeV}$ for a mass of $2 \mathrm{TeV}$. This should be contrasted with, for example, the factor of about 7 increase of the production cross-section that is expected for a $q \bar{q}$ resonance of the same mass. Hence, with the sgoldstino signal hypothesis, also taking into account that the background is mainly due to $q \bar{q}$, one expects the diboson excess to grow significantly faster with the incoming $13 \mathrm{TeV}$ data, with respect to, for instance, a heavy vector signal hypothesis. The different scaling of the signal cross sections with the collider energy could help, in case of discovery, to understand the nature of the new resonance.

Let us end by encouraging the ATLAS and CMS collaborations to provide the efficiencies for all the relevant spin hypotheses of the resonance and the polarizations of the vector bosons in the final state. The fact that the ATLAS analysis [1] only provides the efficiencies for spin-one and -two resonances decaying to longitudinally polarized vector bosons introduces a large uncertainty in our interpretation of the excess. However, we expect this to only amount to a rescaling and possibly a shift of the relevant parameter space region towards slightly lower values of $m_{2}, m_{3}$ and $\sqrt{f}$.

\section{Acknowledgments}

We are thankful to A. Wulzer and F. Zwirner for discussions and comments on the manuscript. We also thank Enrique Kajomovitz for clarifications concerning the ATLAS $Z \gamma$ search at Run I. The work of C. P. is supported by the Swedish Research Council (VR) under the contract 637-2013-475, by IISN-Belgium (conventions 4.4511.06, 4.4505.86 and 
4.4514.08) and by the "Communauté Française de Belgique" through the ARC program and by a "Mandat d'Impulsion Scientifique" of the F.R.S.-FNRS. The work of R.T. was supported by the Italian PRIN no. 2010YJ2NYW_003. R.T. acknowledges CERN hospitality during the completion of this work.

Open Access. This article is distributed under the terms of the Creative Commons Attribution License (CC-BY 4.0), which permits any use, distribution and reproduction in any medium, provided the original author(s) and source are credited.

\section{References}

[1] ATLAS collaboration, Search for high-mass diboson resonances with boson-tagged jets in proton-proton collisions at $\sqrt{s}=8 \mathrm{TeV}$ with the ATLAS detector, JHEP 12 (2015) 055 [arXiv: 1506.00962] [INSPIRE].

[2] CMS collaboration, Search for massive resonances in dijet systems containing jets tagged as $W$ or $Z$ boson decays in pp collisions at $\sqrt{s}=8 \mathrm{TeV}$, JHEP 08 (2014) 173 [arXiv: 1405.1994] [INSPIRE].

[3] H.S. Fukano, M. Kurachi, S. Matsuzaki, K. Terashi and K. Yamawaki, 2 TeV walking technirho at LHC?, Phys. Lett. B 750 (2015) 259 [arXiv: 1506. 03751] [InSPIRE].

[4] J. Hisano, N. Nagata and Y. Omura, Interpretations of the ATLAS diboson resonances, Phys. Rev. D 92 (2015) 055001 [arXiv:1506.03931] [InSPIRE].

[5] D.B. Franzosi, M.T. Frandsen and F. Sannino, Diboson signals via Fermi scale spin-one states, Phys. Rev. D 92 (2015) 115005 [arXiv:1506.04392] [InSPIRE].

[6] K. Cheung, W.-Y. Keung, P.-Y. Tseng and T.-C. Yuan, Interpretations of the ATLAS diboson anomaly, Phys. Lett. B 751 (2015) 188 [arXiv:1506.06064] [INSPIRE].

[7] B.A. Dobrescu and Z. Liu, $W^{\prime}$ boson near 2 TeV: predictions for Run 2 of the LHC, Phys. Rev. Lett. 115 (2015) 211802 [arXiv:1506.06736] [INSPIRE].

[8] J.A. Aguilar-Saavedra, Triboson interpretations of the ATLAS diboson excess, JHEP 10 (2015) 099 [arXiv: 1506.06739] [INSPIRE].

[9] A. Alves, A. Berlin, S. Profumo and F.S. Queiroz, Dirac-fermionic dark matter in $\mathrm{U}(1)_{X}$ models, JHEP 10 (2015) 076 [arXiv:1506.06767] [INSPIRE].

[10] Y. Gao, T. Ghosh, K. Sinha and J.-H. Yu, $\mathrm{SU}(2) \times \mathrm{SU}(2) \times \mathrm{U}(1)$ interpretations of the diboson and $W h$ excesses, Phys. Rev. D 92 (2015) 055030 [arXiv:1506.07511] [InSPIRE].

[11] A. Thamm, R. Torre and A. Wulzer, Composite heavy vector triplet in the ATLAS diboson excess, Phys. Rev. Lett. 115 (2015) 221802 [arXiv:1506.08688] [INSPIRE].

[12] J. Brehmer, J. Hewett, J. Kopp, T. Rizzo and J. Tattersall, Symmetry restored in dibosons at the LHC?, JHEP 10 (2015) 182 [arXiv:1507.00013] [INSPIRE].

[13] Q.-H. Cao, B. Yan and D.-M. Zhang, Simple non-Abelian extensions of the Standard Model gauge group and the diboson excesses at the LHC, Phys. Rev. D 92 (2015) 095025 [arXiv: 1507.00268] [INSPIRE].

[14] G. Cacciapaglia and M.T. Frandsen, Unitarity implications of a diboson resonance in the TeV region for Higgs physics, Phys. Rev. D 92 (2015) 055035 [arXiv: 1507.00900] [INSPIRE]. 
[15] T. Abe, R. Nagai, S. Okawa and M. Tanabashi, Unitarity sum rules, three-site moose model and the ATLAS 2 TeV diboson anomalies, Phys. Rev. D 92 (2015) 055016 [arXiv: 1507.01185] [INSPIRE].

[16] T. Abe, T. Kitahara and M.M. Nojiri, Prospects for spin-1 resonance search at 13 TeV LHC and the ATLAS diboson excess, arXiv:1507.01681 [INSPIRE].

[17] A. Carmona, A. Delgado, M. Quirós and J. Santiago, Diboson resonant production in non-custodial composite Higgs models, JHEP 09 (2015) 186 [arXiv: 1507.01914] [INSPIRE].

[18] B.C. Allanach, B. Gripaios and D. Sutherland, Anatomy of the ATLAS diboson anomaly, Phys. Rev. D 92 (2015) 055003 [arXiv: 1507.01638] [INSPIRE].

[19] C.-W. Chiang, H. Fukuda, K. Harigaya, M. Ibe and T.T. Yanagida, Diboson resonance as a portal to hidden strong dynamics, JHEP 11 (2015) 015 [arXiv:1507.02483] [INSPIRE].

[20] G. Cacciapaglia, A. Deandrea and M. Hashimoto, Scalar hint from the diboson excess?, Phys. Rev. Lett. 115 (2015) 171802 [arXiv:1507.03098] [INSPIRE].

[21] H.S. Fukano, S. Matsuzaki and K. Yamawaki, Conformal barrier for new vector bosons decay to the Higgs, arXiv:1507.03428 [INSPIRE].

[22] V. Sanz, On the compatibility of the diboson excess with a gg-initiated composite sector, arXiv: 1507.03553 [INSPIRE].

[23] C.-H. Chen and T. Nomura, 2 TeV Higgs boson and diboson excess at the LHC, Phys. Lett. B 749 (2015) 464 [arXiv: 1507.04431] [INSPIRE].

[24] Y. Omura, K. Tobe and K. Tsumura, Survey of Higgs interpretations of the diboson excesses, Phys. Rev. D 92 (2015) 055015 [arXiv: 1507.05028] [INSPIRE].

[25] W. Chao, ATLAS diboson excesses from the stealth doublet model, Phys. Lett. $\mathbf{B} 753$ (2016) 117 [arXiv: 1507.05310] [INSPIRE].

[26] L.A. Anchordoqui, I. Antoniadis, H. Goldberg, X. Huang, D. Lüst and T.R. Taylor, Stringy origin of diboson and dijet excesses at the LHC, Phys. Lett. B 749 (2015) 484 [arXiv: 1507.05299] [INSPIRE].

[27] L. Bian, D. Liu and J. Shu, Low scale composite Higgs model and $1.8 \sim 2$ TeV diboson excess, arXiv: 1507.06018 [INSPIRE].

[28] H.M. Lee, D. Kim, K. Kong and S.C. Park, Diboson excesses demystified in effective field theory approach, JHEP 11 (2015) 150 [arXiv:1507.06312] [INSPIRE].

[29] K. Lane and L. Pritchett, Heavy vector partners of the light composite Higgs, Phys. Lett. B 753 (2016) 211 [arXiv: 1507.07102] [INSPIRE].

[30] A.E. Faraggi and M. Guzzi, Extra $Z^{\prime} s$ and $W^{\prime} s$ in heterotic-string derived models, Eur. Phys. J. C 75 (2015) 537 [arXiv:1507.07406] [INSPIRE].

[31] M. Low, A. Tesi and L.-T. Wang, Composite spin-1 resonances at the LHC, Phys. Rev. D 92 (2015) 085019 [arXiv:1507.07557] [INSPIRE].

[32] S.P. Liew and S. Shirai, Testing ATLAS diboson excess with dark matter searches at LHC, JHEP 11 (2015) 191 [arXiv:1507.08273] [INSPIRE].

[33] H. Terazawa and M. Yasue, Excited gauge and Higgs bosons in the unified composite model, arXiv: 1508.00172 [INSPIRE]. 
[34] P. Arnan, D. Espriu and F. Mescia, Interpreting a 2 TeV resonance in $W W$ scattering, arXiv: 1508.00174 [INSPIRE].

[35] C. Niehoff, P. Stangl and D.M. Straub, Direct and indirect signals of natural composite Higgs models, arXiv: 1508.00569 [INSPIRE].

[36] P.S. Bhupal Dev and R.N. Mohapatra, Unified explanation of the eejj, diboson and dijet resonances at the LHC, Phys. Rev. Lett. 115 (2015) 181803 [arXiv: 1508. 02277] [InSPIRE].

[37] S. Fichet, G. von Gersdorff and G. Gersdorff, Effective theory for neutral resonances and a statistical dissection of the ATLAS diboson excess, JHEP 12 (2015) 089 [arXiv:1508.04814] [INSPIRE].

[38] D. Gonçalves, F. Krauss and M. Spannowsky, Augmenting the diboson excess for the LHC Run II, Phys. Rev. D 92 (2015) 053010 [arXiv: 1508.04162] [InSPIRE].

[39] ATLAS collaboration, Search for new phenomena in the dijet mass distribution using pp collision data at $\sqrt{s}=8 \mathrm{TeV}$ with the ATLAS detector, Phys. Rev. D 91 (2015) 052007 [arXiv: 1407.1376] [INSPIRE].

[40] E. Perazzi, G. Ridolfi and F. Zwirner, Signatures of massive sgoldstinos at $e^{+} e^{-}$colliders, Nucl. Phys. B 574 (2000) 3 [hep-ph/0001025] [InSPIRE].

[41] E. Perazzi, G. Ridolfi and F. Zwirner, Signatures of massive sgoldstinos at hadron colliders, Nucl. Phys. B 590 (2000) 287 [hep-ph/0005076] [INSPIRE].

[42] D.S. Gorbunov and N.V. Krasnikov, Prospects for sgoldstino search at the LHC, JHEP 07 (2002) 043 [hep-ph/0203078] [inSPIRE].

[43] I. Antoniadis, E. Dudas and D.M. Ghilencea, Goldstino and sgoldstino in microscopic models and the constrained superfields formalism, Nucl. Phys. B 857 (2012) 65 [arXiv:1110.5939] [INSPIRE].

[44] D. Bertolini, K. Rehermann and J. Thaler, Visible supersymmetry breaking and an invisible Higgs, JHEP 04 (2012) 130 [arXiv:1111.0628] [INSPIRE].

[45] C. Petersson and A. Romagnoni, The MSSM Higgs sector with a dynamical goldstino supermultiplet, JHEP 02 (2012) 142 [arXiv:1111.3368] [INSPIRE].

[46] B. Bellazzini, C. Petersson and R. Torre, Photophilic Higgs from sgoldstino mixing, Phys. Rev. D 86 (2012) 033016 [arXiv:1207.0803] [INSPIRE].

[47] I. Antoniadis and D.M. Ghilencea, Low-scale SUSY breaking and the (s)goldstino physics, Nucl. Phys. B 870 (2013) 278 [arXiv:1210.8336] [InSPIRE].

[48] C. Petersson, A. Romagnoni and R. Torre, Liberating Higgs couplings in supersymmetry, Phys. Rev. D 87 (2013) 013008 [arXiv: 1211.2114] [INSPIRE].

[49] E. Dudas, C. Petersson and P. Tziveloglou, Low scale supersymmetry breaking and its LHC signatures, Nucl. Phys. B 870 (2013) 353 [arXiv:1211.5609] [INSPIRE].

[50] E. Dudas, C. Petersson and R. Torre, Collider signatures of low scale supersymmetry breaking: a Snowmass 2013 white paper, arXiv:1309.1179 [INSPIRE].

[51] K.O. Astapov and S.V. Demidov, Sgoldstino-Higgs mixing in models with low-scale supersymmetry breaking, JHEP 01 (2015) 136 [arXiv:1411.6222] [INSPIRE].

[52] A. Brignole, F. Feruglio and F. Zwirner, Aspects of spontaneously broken $N=1$ global supersymmetry in the presence of gauge interactions, Nucl. Phys. B 501 (1997) 332 [hep-ph/9703286] [INSPIRE]. 
[53] ATLAS collaboration, Search for high-mass diphoton resonances in pp collisions at $\sqrt{s}=8 \mathrm{TeV}$ with the ATLAS detector, Phys. Rev. D 92 (2015) 032004 [arXiv:1504.05511] [INSPIRE].

[54] CMS collaboration, Search for massive resonances decaying into pairs of boosted bosons in semi-leptonic final states at $\sqrt{s}=8 \mathrm{TeV}$, JHEP 08 (2014) 174 [arXiv:1405.3447] [INSPIRE].

[55] ATLAS collaboration, Summary of the searches for squarks and gluinos using $\sqrt{s}=8 \mathrm{TeV}$ pp collisions with the ATLAS experiment at the LHC, JHEP 10 (2015) 054 [arXiv: 1507.05525] [INSPIRE].

[56] CMS collaboration, Search for resonances and quantum black holes using dijet mass spectra in proton-proton collisions at $\sqrt{s}=8 \mathrm{TeV}$, Phys. Rev. D 91 (2015) 052009 [arXiv: 1501.04198] [INSPIRE].

[57] ATLAS collaboration, Search for new resonances in $W \gamma$ and $Z \gamma$ final states in pp collisions at $\sqrt{s}=8 \mathrm{TeV}$ with the ATLAS detector, Phys. Lett. B 738 (2014) 428 [arXiv:1407.8150] [INSPIRE]. 